\title{
SCALAR CURVATURE OF LIE GROUPS
}

\author{
HENG-LUNG LAI AND HUEI-SHYONG LUE ${ }^{1}$
}

\begin{abstract}
ABSTRACr. In this paper, we prove the following theorem: If $G$ is a connected Lie group, then $G$ admits left invariant metric of positive scalar curvature if and only if the universal covering space $\tilde{G}$ of $G$ is not homeomorphic to the Euclidean space.
\end{abstract}

1. Introduction. In [8], Yamabe claimed that a smooth compact Riemannian manifold of dimension at least 3 can be conformally deformed to a metric of constant scalar curvature. Although Yamabe's proof turned out to be defective, his work has led to important work on the study of scalar curvature. In [1], [2], Aubin and Eliasson showed that on a smooth compact manifold of dimension at least 3 there admits a metric of strictly negative scalar curvature. However there does not always exist positive scalar curvature on a manifold. Hitchin [5] showed that an exotic sphere which does not bound a spin manifold cannot admit a metric of positive scalar curvature. Schoen and Yau [7] showed that if $M$ is an orientable three dimensional manifold with nonnegative scalar curvature, and if $\pi_{1}(M)$ contains a subgroup which is abstractly isomorphic to the fundamental group of a compact surface of genus at least one, then $M$ is isometric to the three dimensional flat torus. The result is further generalized by Schoen and Yau to dimensions not greater than 7. Gromov and Lawson generalized to the special case of spin manifolds of all dimensions. As to a Lie group with left invariant metric, Milnor [6] showed that a solvable Lie group with left invariant metric is either flat or has strictly negative scalar curvature. Wallach gave a sufficient condition for the existence of positive scalar curvature. Wallach's theorem showed that if the universal covering space of a Lie group is not homeomorphic to Euclidean space, then the Lie group admits a left invariant metric of positive scalar curvature. Milnor conjectured that these are the only possible ways to obtain positive scalar curvature on a Lie group with left invariant metric. In this paper, we give an affirmative answer to the above conjecture and prove the following.

TheOREM. If $G$ is a connected Lie group, then $G$ admits a left invariant metric of positive scalar curvature if and only if the universal covering space $\tilde{G}$ of $G$ is not homeomorphic to the Euclidean space.

Notice that the result by Aubin and Eliasson demonstrated the topological insignificance of the negative scalar curvature, while our result indicates the

Received by the editors December 10, 1979.

1980 Mathematics Subject Classification. Primary 22E99, 57 E99.

${ }^{1}$ Under partial support by N.S.C. of R.O.C. 
topology of a Lie group played a crucial role as to the existence of positive scalar curvature on Lie groups with metric invariant by left translations.

The authors would like to express their appreciation to the referee for bringing their attention to the results obtained by Schoen-Yau, Gromov-Lawson.

2. Preliminaries. In this section, we define some basic notions which are necessary for our purpose. For more details, we refer to [4].

Let $G$ be a Lie group, the associated Lie algebra is denoted by $\mathbb{S}$, consisting of smooth vector fields which are invariant under left translations. If $e_{1}, \ldots, e_{n}$ is a basis of $\mathbb{B}$, its bracket product is given by

$$
\left[e_{i}, e_{j}\right]=\sum_{k=1}^{n} c_{i j}^{k} e_{k}
$$

where the structure constants $\left\{c_{i j}^{k}\right\}$ satisfying

$$
c_{i j}^{k}=-c_{j i}^{k}, \quad \sum_{l}\left(c_{i j}^{l} c_{l k}^{m}+c_{j k}^{l} c_{l i}^{m}+c_{k i}^{l} c_{l j}^{m}\right)=0 .
$$

To a metric $\langle$,$\rangle on G$, there exists a uniquely defined Riemannian connection $\nabla$, which assigns to each pair of smooth vector fields $x, y$, a smooth vector field $\nabla_{x} y$. This is called the covariant derivative of $y$ in the direction $x$. For our purpose, we specify the characterizing properties of $\nabla$ as follows:

$$
\begin{aligned}
& \nabla_{x} y-\nabla_{y} x=[x, y] \quad \text { (torsion free), } \\
& \left\langle\nabla_{x} y, z\right\rangle+\left\langle y, \nabla_{x} z\right\rangle=x\langle y, z\rangle .
\end{aligned}
$$

If $\langle$,$\rangle is a metric on G$ which is invariant under left translations, then to each pair of smooth left invariant vector fields $x$ and $y, \nabla_{x} y$ is also a left invariant vector field and satisfies $\left\langle\nabla_{x} y, z\right\rangle+\left\langle y, \nabla_{x} z\right\rangle=0,2\left\langle\nabla_{x} y, z\right\rangle=\langle[x, y], z\rangle-\langle[y, z]$, $x\rangle+\langle[z, x], y\rangle$ for all $x, y, z$ in $\mathbb{S}$.

The Riemannian curvature tensor $R$ associates to each pair of smooth vector fields $x$ and $y$ the linear transformation

$$
R_{x y}=\nabla_{x} \nabla_{y}-\nabla_{y} \nabla_{x}-\nabla_{[x, y]}
$$

Evidently $R_{x y}=-R_{y x}$. The transformation $R_{x y}$ is always skew-adjoint, that is, $\left\langle R_{x y} z, w\right\rangle+\left\langle z, R_{x y} w\right\rangle=0$.

If $x$ and $y$ are orthonormal, the number $k(x, y)=\left\langle R_{x y} y, x\right\rangle$ is called the sectional curvature of the plane determined by $x$ and $y$. For smooth vector fields $x$ and $y$, define $r(x, y)=\operatorname{Tr}\left(z \rightarrow R_{z x} y\right)$ called the Ricci tensor of $x$ and $y$. Sometimes it is more convenient to work with the selfadjoint operator $\hat{r}$, called the Ricci transformation, defined by

$$
\hat{r}(x)=\sum_{i=1}^{n} R_{x e_{i}} e_{i} ;
$$

then we have $\langle\hat{r}(x), y\rangle=r(x, y)$. The eigenvalues $\lambda_{1}, \lambda_{2}, \ldots, \lambda_{n}$ of $\hat{r}$ are called the principal Ricci curvatures.

For unit tangent vector $x, r(x)=r(x, x)$ is called the Ricci curvature in the direction $x . \rho=\lambda_{1}+\lambda_{2}+\cdots+\lambda_{n}$ is called the scalar curvature.

It was proved in [6] that to every left invariant metric on a Lie group with Lie 
algebra $\operatorname{sl}(2 ; \mathbf{R})$, the scalar curvature is always strictly negative. For our later reference, we shall reproduce the proof here as an example.

EXAMPLE. To every left invariant metric on a Lie group with Lie algebra sl(2; R), there is an orthonormal basis $e_{1}, e_{2}, e_{3}$ in $\operatorname{sl}(2 ; \mathbf{R})$ such that

$$
\left[e_{1}, e_{2}\right]=\lambda_{3} e_{3}, \quad\left[e_{2}, e_{3}\right]=\lambda_{1} e_{1},\left[e_{3}, e_{1}\right]=\lambda_{2} e_{2} \text {. }
$$

If we define $\mu_{i}=\frac{1}{2}\left(\lambda_{1}+\lambda_{2}+\lambda_{3}\right)-\lambda_{i}(i=1,2,3)$, then the associated Riemannian connection $\nabla$ satisfies

$$
\begin{gathered}
\nabla_{e_{i}} e_{i}=0 \text { for } i=1,2,3 . \\
\nabla_{e_{1}} e_{2}=\mu_{1} e_{3}, \quad \nabla_{e_{2}} e_{3}=\mu_{2} e_{1}, \quad \nabla_{e_{3}} e_{1}=\mu_{3} e_{2} .
\end{gathered}
$$

Since $\hat{r}(x)=\sum_{i=1}^{3} R_{x e_{i}} e_{i}$, we have $\hat{r}\left(e_{1}\right)=2 \mu_{2} \mu_{3} e_{1}, \quad \hat{r}\left(e_{2}\right)=2 \mu_{1} \mu_{3} e_{2}, \quad \hat{r}\left(e_{3}\right)=$ $2 \mu_{1} \mu_{2} e_{3}$. Hence the principal Ricci curvatures are $2 \mu_{1} \mu_{2}, 2 \mu_{1} \mu_{3}$ and $2 \mu_{2} \mu_{3}$. So the scalar curvature is given by $\rho=2\left(\mu_{1} \mu_{2}+\mu_{2} \mu_{3}+\mu_{3} \mu_{1}\right)$. It is further proved in [6] that $\rho<0$.

As to the Lie group theoretical aspects of our works, the results described below are more or less well known. We include the results for the sake of self-containedness. For more detailed discussion, we refer to [4].

Consider the Levi-decomposition of Lie algebra $\& S=s \oplus r$ where $r$ is the radical of $\mathbb{S}$ and $s$ is a maximal semisimple subalgebra of $\&$. Denote by $\tilde{G}$ the universal covering group of $G$. Denote by $S$ (resp. $R$ ) the subgroup of $\tilde{G}$ corresponding to $S$ (resp. $r$ ). Then $S$ and $R$ are closed and simply connected. Furthermore, $\tilde{G}=S \times$ $R$ (homeomorphism), $S \cap R=\{e\}$.

If we write $s$ as the direct sum of simple ideals $s=s_{1} \oplus \ldots \oplus s_{l}$ then check the list in [4], or the table in [3], the only simple Lie algebras with maximal compact subalgebra being abelian can only be $A I(2), A I I I(1,1), B D I(2,1)$, or $C I(1)$, but they are all isomorphic to $\mathrm{sl}(2 ; \mathbf{R})$.

Hence if $\tilde{G}$ is homeomorphic to Euclidean space, then as a vector space,

$$
\text { (S) }=\operatorname{sl}(2 ; \mathbf{R}) \oplus \cdots \oplus \operatorname{sl}(2 ; \mathbf{R}) \oplus r=\operatorname{sl}(2 ; \mathbf{R}) \oplus 3 \oplus r
$$

where 3 is the direct sum of $(l-1)$ copies of $\operatorname{sl}(2 ; \mathbf{R})$.

For $x=x_{1}+y_{1}+z_{1} \in \mathbb{S}, y=y_{2}+z_{2} \in 3 \oplus r$ (where $x_{i} \in \operatorname{sl}(2 ; \mathbf{R}), y_{i} \in 3$, $\left.z_{i} \in r\right)$,

$$
[x, y]=\left[x, z_{2}\right]+\left[x_{1}+y_{1}, y_{2}\right]+\left[z_{1}, y_{2}\right] \in 3 \oplus r .
$$

This is because $r$ is an ideal and 3 is an ideal in $s$. Thus $3 \oplus r$ is an ideal of $\mathbb{S}$. Summing up our discussion, we have the following.

Proposition. $\tilde{G}$ is homeomorphic to Euclidean space if and only if its Lie algebra (S) is either solvable or can be decomposed as $\mathbb{B}=\operatorname{sl}(2 ; \mathbf{R}) \oplus \mathfrak{A}$, where $\mathfrak{A}$ is an ideal of (S, $\mathfrak{A}=s^{\prime} \oplus r, s^{\prime}$ is the direct sum of copies of $\mathrm{sl}(2 ; \mathbf{R})$ as ideals, and $r$ is the radical of \&S.

3. Proof of the theorem. If $G$ is solvable, then in [6], Milnor has proved that every left invariant metric is either flat or has strictly negative scalar curvature.

From now on, we may assume that the Lie group $G$ is not solvable and the universal covering space is homeomorphic to Euclidean space. 
Given any left invariant metric $\langle$,$\rangle on G$, consider the decomposition of its Lie algebra $\mathbb{S}$ as in the proposition, $\mathbb{S}=\operatorname{sl}(2 ; \mathbf{R}) \oplus \mathfrak{A}$. The orthogonal complement of $\mathfrak{A}$ is $\mathfrak{A}^{\perp}=\{x \in \mathfrak{S} \mid\langle x, y\rangle=0 \forall y \in \mathfrak{A}\} . \mathfrak{A}^{\perp} \simeq \operatorname{sl}(2 ; \mathbf{R})$ as Lie algebra. Hence we may assume that the decomposition $\mathbb{S}=\operatorname{sl}(2 ; \mathbf{R}) \oplus \mathfrak{A}$ is an orthogonal direct sum.

Let $\left\{e_{1}, e_{2}, e_{3}, f_{i}\right\}_{i=1, \ldots, m}$ be an orthonormal basis for \&S such that $\left\{e_{1}, e_{2}, e_{3}\right\}$ is an orthonormal basis for $\operatorname{sl}(2 ; \mathbf{R})$ as chosen in the example, $\left[e_{1}, e_{2}\right]=\lambda_{3} e_{3},\left[e_{2}, e_{3}\right]$ $=\lambda_{1} e_{1},\left[e_{3}, e_{1}\right]=\lambda_{2} e_{2}$.

We denote by the induced linear transformation ad $e_{i}$ on $\mathfrak{A}$ as $L_{i}$. Notice that in sl $(2 ; \mathbf{R}), \lambda_{1}, \lambda_{2}, \lambda_{3}$ are not zero; hence the above bracket product formula implies that

$$
\left[L_{1}, L_{2}\right]=\lambda_{3} L_{3}, \quad\left[L_{2}, L_{3}\right]=\lambda_{1} L_{1}, \quad\left[L_{3}, L_{1}\right]=\lambda_{2} L_{2}
$$

From this, we know $\operatorname{tr} L_{i}=0$ for $i=1,2,3$.

Denote by $L_{i}^{*}$ the adjoint linear transformation of $L_{i}$ on $\mathfrak{A}$, that is, $\left\langle L_{i} x, y\right\rangle=$ $\left\langle x, L_{i}^{*} y\right\rangle$, for $x, y$ in $\mathfrak{A}$. Put

$$
S_{i}=\frac{1}{2}\left(L_{i}+L_{i}^{*}\right), \quad A_{i}=\frac{1}{2}\left(L_{i}-L_{i}^{*}\right) .
$$

Denote by $\nabla$ the Riemannian connection associated with given metric on $G, \bar{\nabla}$ (resp. $\left.\nabla^{\prime}\right)$ the Riemannian connection of the induced metric on $\mathfrak{A}$ (resp. sl(2; R)). A simple but tedious computation yields the formula $\nabla_{e_{i}} e_{j}=\nabla_{e_{i}}^{\prime} e_{j}$, where $\nabla_{e_{i}}^{\prime} e_{j}$ is as given in the example of $\$ 2$.

$$
\begin{aligned}
& \nabla_{e_{i}} f_{j}=A_{i}\left(f_{j}\right), \quad \nabla_{f_{j}} e_{i}=-S_{i}\left(f_{j}\right), \\
& \nabla_{f_{i}} f_{j}=\sum_{k=1}^{3}\left\langle S_{k} f_{i}, f_{j}\right\rangle e_{k}+\bar{\nabla}_{f_{i}} f_{j} .
\end{aligned}
$$

From this, we can compute the Ricci curvature. By the same way, we denote $\bar{r}$ (resp. $\left.r^{\prime}\right)$ the Ricci curvature of the induced metric on $\mathfrak{A}$ (resp. $\mathrm{sl}(2 ; \mathbf{R})$ ).

$$
\begin{aligned}
& r\left(f_{j}\right)=\left\langle\sum_{i=1}^{3}\left(A_{i} S_{i}-S_{i} A_{i}\right) f_{j}, f_{j}\right\rangle+\bar{r}\left(f_{j}\right), \\
& r\left(e_{i}\right)=-T r S_{i}^{2}+r^{\prime}\left(e_{i}\right)
\end{aligned}
$$

so the scalar curvature is given by

$$
\rho=\sum_{i=1}^{3} r\left(e_{i}\right)+\sum_{i=1}^{m} r\left(f_{i}\right)=\rho^{\prime}+\bar{\rho}-\sum_{i=1}^{3} \operatorname{tr} S_{i}^{2}
$$

where $\bar{\rho}$ (resp. $\left.\rho^{\prime}\right)$ is the scalar curvature on $\mathfrak{A}$ (resp. $\mathrm{sl}(2 ; \mathbf{R})$ ).

In [6], it has been shown that $\rho^{\prime}<0$ for every left invariant metric on Lie group with Lie algebra $\operatorname{sl}(2 ; \mathbf{R})$. We claim that $\bar{\rho}<0$. We prove this by induction on the number $l$ of copies $\operatorname{sl}(2 ; \mathbf{R})$ appearing as direct factors in $\mathbb{S}$. For $l=1, \mathfrak{A}$ is solvable; hence either the metric is flat or $\bar{\rho}<0$ as in [6]. Hence by induction we have $\bar{\rho}<0 . S_{i}$ is selfadjoint for $i=1,2,3$; hence $\operatorname{tr} S_{i}^{2}>0$. So we conclude that $\rho<0$ and the theorem is proved. 


\section{BIBLIOGRAPHY}

1. T. Aubin, Metriques riemanniennes et curbure, J. Differential Geometry 4 (1970), 383-424.

2. H. Eliasson, On variation of metric, Math. Scand. 29 (1971), 317-327.

3. M. Goto, Lattices of subalgebras of real Lie algebras, J. Algebra 11 (1969), 6-24.

4. S. Helgason, Differential geometry, Lie groups and symmetric spaces, Academic Press, New York, 1978.

5. N. Hitchin, Harmonic spinors, Advances in Math. 14 (1974), 1-55.

6. J. Milnor, Curvature of left invariant metric on Lie groups, Advances in Math. 21 (1976), 293-329.

7. R. Schoen and S. T. Yau, Existence of incompressible minimal surfaces and the topology of three dimensional manifolds with nonnegative scalar curvature, Ann. of Math. (2) 110 (1979), 127-142.

8. H. Yamabe, On a deformation of Riemannian structures on a compact manifold, Osaka J. Math. 12 (1960), 21-37.

Department of Mathematics, National Tsing Hua University, Hsinchu, Tatwan, Republic of ChIna 